\title{
MRI Image Classification of Brain Tumor Using Deep Neural Network and Deployment Using Web Framework
}

\author{
Sumithra $\mathrm{M}^{\mathrm{a}, 1}$, Shruthi $\mathrm{S}^{\mathrm{b}}$, SmithiRam ${ }^{\mathrm{b}}$, Swathi $\mathrm{S}^{\mathrm{b}}$, Deepika $\mathrm{T}^{\mathrm{b}}$ \\ ${ }^{a}$ Associate Professor, Panimalar Engineering College, Chennai \\ ${ }^{b} U G$ scholar, Panimalar Engineering College, Chennai
}

\begin{abstract}
A brain tumor is a mass or growth of abnormal cells in our brain. Many different types of brain tumors exist. Some brain tumors are noncancerous (benign), and some brain tumors are cancerous (malignant). Brain tumors can begin in your brain (primary brain tumors), or cancer can begin in other parts of your body and spread to your brain (secondary, or metastatic, brain tumors). Brain tumor treatment options depend on the type of brain tumor you have, as well as its size and location. The classification of brain tumors is performed by biopsy, which is not usually conducted before definitive brain surgery. The improvement of technology and machine learning can help radiologists in tumor diagnostics without invasive measures. A machine-learning algorithm that has achieved substantial results in image classification is the convolution neural network $(\mathrm{CNN})$. It is predicted that the success of the obtained results will increase if the CNN method is supported by adding extra feature extraction methods and classify successfully brain tumor normal and abnormal image.
\end{abstract}

Keywords:brain tumor, deep learning, Tensor Flow, CNN

\section{Introduction}

The classification of brain tumors is performed by biopsy, which is not usually conducted before definitive brain surgery. The improvement of technology and machine learning can help radiologists in tumor diagnostics without invasive measures[6]. A machine-learning algorithm that has achieved substantial results in image segmentation and classification is the convolutional neural network (CNN). The classification was performed using a T1-weighted contrast-enhanced MRI image database which contains three tumor types. As input, we used whole images, so it was not necessary to perform any preprocessing or segmentation of the tumors[7],

\footnotetext{
${ }^{1}$ Sumithra M, Associate Professor, Panimalar Engineering College, Chennai, Email: sumithram.id@gmail.com
} 
Samples of more number of images are collected that comprised of different classes such as normal and abnormal. Different number of images is collected for each class that was classified into input images. we proposed a Deep Learning (DL) based brain tumor prediction method to prevent disease by cultivating. The DL method used in the study is the Convolution Neural Network $(\mathrm{CNN})[10]$. It is predicted that the success of the obtained results will increase if theCNN method is supported by adding extra feature [8] extraction methods and classify successfully brain tumor. To deployment these processes by show the prediction result in local host web application.

\section{Architecture}

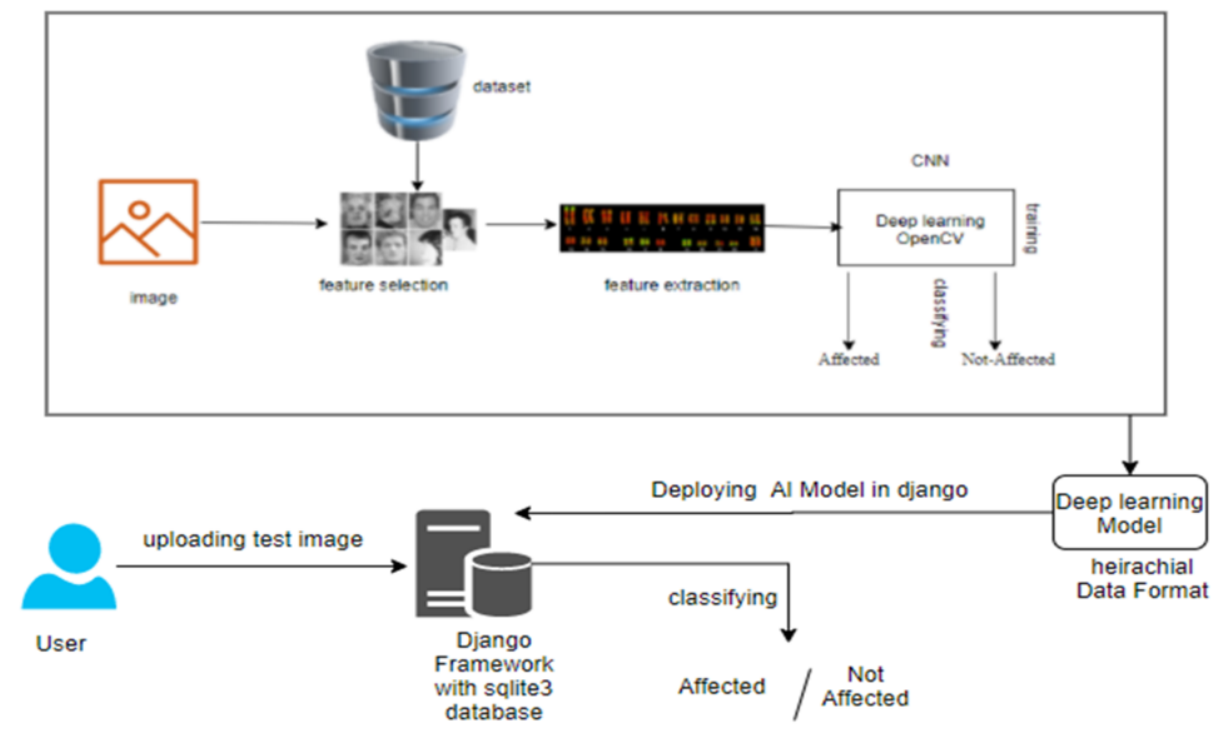

Figure 1. Architecture Diagram

The images which are both tumorous and non-tumorous are trained in the dataset using deep learning algorithm. When the user uploads a new image, it can easily identify whether the image is tumorous or not using the trained dataset. It is done in Django framework with slight 3 database. The user uploads the image in .h5 format (hierarchical data format[9]) which is the supported format in the python framework.

\section{Methodology}

\subsection{Loading the given Brain Tumor image (module01)}

The data set is imported using keras preprocessing image data generator function also we create size, rescale, range, zoom range, horizontal flip. Then we import our image dataset from folder through the data generator function. Then we make folders as train, test, and validation also we set target size, batch size and class-mode from this function we have to train of brain tumor. 


\subsection{Training the given Tumor image using Convolution Neural Network(module02)}

The dataset that is the images brain tumor and normal is collected and the LeNet CNN is applied on the model and the accuracy and graph is collected.

\subsection{Working of the Algorithm and .h5 file generation (module03)}

In this module we train the neural network by giving the images by giving more epochs to train better and the .h5 file (Hierarchical Data Format) is generated and the model is loaded and is tested by giving input.

\subsection{Deployment of brain tumor model in Django Framework and predicting output (module04)}

In this module we create a web application using Django framework by using sqlite 3 database.Our .h5 file is deployed in the web application here the image is uploaded and after uploading the model predicts whether the giving image has tumor or not.

\section{Conclusion}

It focused how image from given dataset (trained dataset) in field and past data set used predict the pattern of brain tumor using $\mathrm{CNN}$ model. This brings some of the following insights about tumor prediction. We had applied different type of CNN compared the accuracy and saw that LeNet makes better classification and the .h5 file is taken from there and that is deployed in Django framework for better user interface.

\section{References}

[1] MirceaGurbin, MihaelaLascu, Dan Lascu .Tumor Detection and Classification of MRIBrain Image using Different Wavelet Transforms and Support Vector Machines. 2019 42ndProcessing (TSP), Budapest,Hungary, 2019, pp. 505-508.

[2] M. Rezaei, H. Yang, C. Meinel .Instance Tumor Segmentation using Multitask Convolutional Neural Network.2018 International Joint Conference on Neural Networks (IJCNN), Rio de Janeiro, 2018, pp. $1-8$.

[3] HarshiniBadisa, MadhaviPolireddy, Aslam Mohammed.CNN Based Brain Tumor Detection, International Journal of Engineering and Advanced Technology (IJEAT), Volume-8 Issue-4, April 2019 , pp. $1731-1734$.

[4] MB Bramarambika, Seshashayee .Brain Tumor Detection and Identification Using Histogram Method. International Journal of Innovative Technology and Exploring Engineering (IJITEE), Volume-8 Issue10, August 2019, pp. $3517-3521$.

[5] TonmoyHossain, FairuzShadmaniShishir, Mohsena Ashraf, MD Abdullah Al Nasim,Faisal Muhammad Shah.Brain Tumor Detection Using Convolutional Neural Network. 1st International Conference on Advances in Science, Engineering and Robotics Technology 2019(ICASERT 2019), Dhaka, Bangladesh, 2019, pp. 1-6.

[6] Sumithra.M and Malathi.S.Segmentation Of Different Modalitites Using Fuzzy K-Means And Wavelet ROI.International Journal Of Scientific \& Technology Research, Volume 8, Issue 11, pp. 996-1002, November 2019. (18302 oct excel scopus list)

[7] Sumithraand.M, Malathi.S .A Brief Survey on Multi Modalities Fusion.Lecture Notes on Data Engineering and Communications Technologies, 35, pp. 1031-1041,2020.

[8] Sumithra.M and Malathi.S .Modified Global Flower Pollination Algorithm-based image fusion for medical diagnosis using computed tomography and magnetic resonance imaging. International Journal of Imaging Systems and Technology.Vol. 31, Issue No.1, pp. 223-235, 2021. 
[9] Sumithra.M and Malathi.S .A survey on Medical Image Segmentation Methods with Different Modalitites. International Journal of Engineering Research and Technology (IJERT) - Volume 6 Issue 2, Mar 2018.

[10] Sumithra.M and Malathi.S .A Survey of Brain Tumor Segmentation Methods with Different Image Modalitites. International Journal of Computer Science Trends and Technology (IJCST) - Volume 5 Issue 2, Mar - Apr 2017.

[11] Ambeth Kumar.V.D et.al .A Comparative Study of Fuzzy Evolutionary Techniques for Footprint Recognition and Performance Improvement using Wavelet based Fuzzy Neural Network.for the International Journal of Computer Applications in Technology, Vol.48, No.2,pp.95 - 105, [ ttp://dx.doi.org/10.1504/IJCAT.2013.056016]

[12] Ambeth Kumar.V.D et.al .Gender Identification Using a Speech Dependent Voice Frequency Estimation System.International Journal of Computer Science and Information Security,, Vol 14, No: 11, PP: 1025-1034, 2016.

[13] Venkatesh.J, Ambeth Kumar.V.D.Advanced Filter and Minutia Matching For Fingerprint Recognition. in the month of January for International Journal of Science and Research (IJSR), Volume 2 Issue 1 January 2013

[14] Ambeth Kumar.V.D, Dr.M.Ramakrishan and G.Jagdeesh (2012).A Discrete correlation of Footprint Image aforementioned to Recognition. in the month of March for the 1sd National Conference on Future Computing 2012 (FUCOMPU),March , 2012.Pondichery University , (karaikal Brach) Karaikal

[15] Ambeth Kumar.V.D et.al..Performance Improvement Using an Automation System for Segmentation of Multiple Parametric Features Based on Human Footprint.for the Journal of Electrical Engineering \& Technology , vol. 10, no. 4, pp.1815-1821 , 2015. [http://dx.doi.org/10.5370/JEET.2015.10.4.1815]

[16] Ambeth Kumar.V.D and Ramakrishan.M(2011).Web Cam Motion Detection Surveillance System Using Temporal Difference and Optical Flow Detection with Multi Alerts.(CCIS) Journal (Springer) Volume 147, Part 3,pp: 214-221, 2011, April 2011 and the paper was published in the International Journal. [Impact Factor: 0.247].[DOI: 10.1007/978-3-642-20573-6_34]

[17] Ruphitha.S.V et.al.Management of Major Postpartum Haemorrhage by using Zigbee protocol - A Review. 2021 6th International Conference on Inventive Computation Technologies (ICICT) (DOI: 10.1109/ICICT50816.2021.9358757 )

[18] M. Indhumathi et.al .Healthcare Management of Major Cardiovascular Disease-A review. 2021 6th International Conference on Inventive Computation Technologies (ICICT), (DOI: 10.1109/ICICT50816.2021.9358519)

[19] Sabarinathan.K et.al . Machine Maintenance Using Augmented Reality. 3rd International Conference on Communication and Electronics Systems (ICCES), 2018. (DOI: 10.1109/CESYS.2018.8723900)

[20] R. Subha Shini et.al., " Recurrent Neural Network based Text Summarization Techniques by Word Sequence Generation",IEEE International Conference on Inventive Computation Technologies (ICICT), 2021, DOI: 10.1109/ICICT50816.2021.9358764 